\title{
NEW INVERSION FORMULAS FOR RADON TRANSFORMS ON AFFINE GRASSMANNIANS
}

\author{
BORIS RUBIN AND YINGZHAN WANG*
}

\begin{abstract}
We obtain new inversion formulas for the Radon transform and the corresponding dual transform acting on affine Grassmann manifolds of planes in $\mathbb{R}^{n}$. The consideration is performed in full generality on continuous functions and functions belonging to $L^{p}$ spaces.
\end{abstract}

\section{INTRODUCTION}

This article is a continuation and generalization of our previous work [16] devoted to integral geometry on line bundles. Let $G(n, k)$ and $G\left(n, k^{\prime}\right)$ be a pair of the affine Grassmann manifolds of $k$-dimensional and $k^{\prime}$-dimensional non-oriented planes in $\mathbb{R}^{n}$, respectively. We suppose that $0<k<k^{\prime}<n$. The excluded case $k=0$ formally corresponds to points in $\mathbb{R}^{n}$. Given sufficiently good functions $f$ on $G(n, k)$ and $\varphi$ on $G\left(n, k^{\prime}\right)$, we consider the following integral transforms

$$
(R f)(\zeta)=\int_{\tau \subset \zeta} f(\tau) d_{\zeta} \tau, \quad\left(R^{*} \varphi\right)(\tau)=\int_{\zeta \supset \tau} \varphi(\zeta) d_{\tau} \zeta
$$

the integration being performed with respect to the corresponding canonical measures. The first integral is called the Radon transform of $f$ and denotes integration over all $k$-planes $\tau$ in the $k^{\prime}$-plane $\zeta$. The second one is called the dual Radon transform of $\varphi$ and integrates over all $k^{\prime}$-planes $\zeta$ containing the $k$-plane $\tau$.

Our goal is to find explicit inversion formulas for these transforms in possibly wide classes of functions. The problem is not new. Similar problems for compact Grassmann manifolds $G_{n, k}, G_{n, k^{\prime}}$ of $k$-dimensional and $k^{\prime}$-dimensional linear subspaces of $\mathbb{R}^{n}$ were studied by many authors, including I.M. Gelfand, M.I. Graev, Z.Ya. Shapiro, R. Roşu,

2010 Mathematics Subject Classification. Primary 44A12; Secondary 47G10.

Key words and phrases. Radon transforms, Grassmann manifolds, Funk transform, Erdélyi-Kober operators.

*Y. Wang was supported by NNSF of China (Grant Nos. 10471040). 
E.E. Petrov, E.L. Grinberg, F. Gonzalez, T. Kakehi, G. Zhang: see $[6,18]$, and references therein.

The noncompact case is essentially more complicated. Different approaches to the study of operators (1.1) are known. M.I. Graev [5] parametrized planes in $\mathbb{R}^{n}$ by matrices and obtained an inversion formula for $R f$ in the so-called local case (when $k^{\prime}-k$ is even) by making use of differential forms and kappa operators. Another inversion formula for $R f$ if $k^{\prime}-k$ is even was suggested by F. Gonzalez and T. Kakehi [3] who used the corresponding Lie algebra language and the Fourier transform techniques. In both publications only smooth rapidly decreasing functions $f$ were considered. One should also mention the paper by Strichartz [17] who developed $L^{2}$ harmonic analysis on Grassmannian bundles.

A completely different approach to operators (1.1) was suggested by the first-named co-author in [10]. The key idea of [10] is to use a certain analogue of the stereographic projection to express (1.1) through the similar operators on compact Grassmannians. The latter can be studied using the tools developed in [6]. This approach enables one to obtain inversion formulas for both $R$ and $R^{*}$ in the framework of Lebesgues spaces for arbitrary $k^{\prime}-k>0$ provided that these operators are injective.

The $L^{p}$-theory of the operators (1.1) is of independent interest. The boundedness of these operators in $L^{p}$ spaces with power weights was studied in [13].

Aim of the Work and Main Results: It is known $[8,15]$ that in the case $k=0$, when $R f$ becomes the classical Radon-John transform, the inversion of $R$ can be performed directly, without using stereographic projection. Note also that the use of the stereographic projection makes all formulas more complicated because of inevitable weight factors. We wonder, if there is a direct way to invert the operators (1.1) under possibly minimal assumptions for functions $f$ and $\varphi$.

New results in this direction are obtained in the present paper. In particular, we show that inversion of (1.1) can be reduced to consecutive inversion of certain Radon-John transforms over lower dimensional planes and Funk-Radon transforms on compact Grassmannians. These 'simpler' transforms can be inverted by known tools; see, e.g., $[6,8,11,14]$ and references therein. New subclasses of the so-called quasi-radial functions, that serve as Grassmannian generalizations of radial functions on $\mathbb{R}^{n}$ and on which operators (1.1) factorize into the tensor product of known integral geometrical objects, are introduced. 
The paper is organized as follows. Section 2 contains necessary background related to Radon-like transforms (in affine and compact settings) and Erdélyi-Kober fractional integrals. These facts are applied in Sections 3 and 4 to inversion of the operators (1.1).

\section{Preliminaries}

2.1. Notation. Let $G(n, k)$ be the affine Grassmann manifold of all non-oriented $k$-dimensional planes in $\mathbb{R}^{n}, 0<k<n$. We denote by $G_{n, k}$ the compact Grassmann manifold of all $k$-dimensional linear subspaces of $\mathbb{R}^{n}$. Each plane $\tau \in G(n, k)$ is parameterized by the pair $(\xi, u)$, where $\xi \in G_{n, k}$ and $u \in \xi^{\perp}$, the orthogonal complement to $\xi$ in $\mathbb{R}^{n}$. We denote by $|\tau|$ the Euclidean distance of $\tau \equiv \tau(\xi, u) \in G(n, k)$ to the origin of $\mathbb{R}^{n}$. Clearly, $|\tau|=|u|$ (the Euclidean norm of $u$ ). The manifold $G(n, k)$ will be endowed with the product measure $d \tau=d \xi d u$, where $d \xi$ is the $O(n)$-invariant probability measure on $G_{n, k}$ and $d u$ denotes the usual volume element on $\xi^{\perp}$. We use the notation $C_{\mu}(G(n, k))$ for the space of continuous function $f$ on $G(n, k)$ satisfying $f(\tau)=O\left(|\tau|^{-\mu}\right), \mu \in \mathbb{R}$. The notation $C_{\mu}\left(\mathbb{R}^{n}\right)$ for the space of continuous functions on $\mathbb{R}^{n}$ has a similar meaning. We also denote

$$
L_{\lambda}^{1}(G(n, k))=\left\{f: \int_{G(n, k)} \frac{|f(\tau)| d \tau}{(1+|\tau|)^{\lambda}}<\infty\right\} .
$$

In the following, $S^{n-1}=\left\{x \in \mathbb{R}^{n}:|x|=1\right\}$ is the unit sphere in $\mathbb{R}^{n}$. For $\theta \in S^{n-1}, d \theta$ stands for the surface element on $S^{n-1} ; \sigma_{n-1}=$ $2 \pi^{n / 2} / \Gamma(n / 2)$ is the surface area of $S^{n-1}$. We set $d_{*} \theta=d \theta / \sigma_{n-1}$ for the normalized surface element on $S^{n-1}$.

For $k^{\prime}>k$ and $\eta \in G_{n, k^{\prime}}$, we denote by $G_{k}(\eta)$ the Grassmann manifold of all $k$-dimensional linear subspaces of $\eta ; e_{1}, \ldots, e_{n}$ are the coordinate unit vectors in $\mathbb{R}^{n}$. Given $0<k<k^{\prime}<n$, we use the following notations for the coordinate planes:

$$
\begin{gathered}
\mathbb{R}^{k}=\mathbb{R} e_{1} \oplus \cdots \oplus \mathbb{R} e_{k}, \quad \mathbb{R}^{k^{\prime}}=\mathbb{R} e_{1} \oplus \cdots \oplus \mathbb{R} e_{k^{\prime}}, \\
\mathbb{R}^{k^{\prime}-k}=\mathbb{R} e_{k+1} \oplus \cdots \oplus \mathbb{R} e_{k^{\prime}}, \quad \mathbb{R}^{n-k}=\mathbb{R} e_{k+1} \oplus \cdots \oplus \mathbb{R} e_{n} . \\
\mathbb{R}^{n-k^{\prime}}=\mathbb{R} e_{k^{\prime}+1} \oplus \cdots \oplus \mathbb{R} e_{n} .
\end{gathered}
$$

The letter $c$ stands for a constant that can be different at each occurrence; $[\alpha]$ denotes the integer part of the real number $\alpha$. All integrals are understood as Lebesgue integrals, unless otherwise stated. We say that an integral under consideration exists in the Lebesgue sense if it is finite when all functions under the sign of integration are replaced by their absolute values. 


\subsection{The Radon Transforms for a Pair of Affine Grassmanni-}

ans. Let $G(n, k)$ and $G\left(n, k^{\prime}\right)$ be a pair of affine Grassmann manifolds of $k$-planes $\tau$ and $k^{\prime}$-planes $\zeta$ in $\mathbb{R}^{n}$, respectively; $1 \leq k<k^{\prime} \leq n-1$. We write

$$
\tau \equiv \tau(\xi, u) \in G(n, k), \quad \zeta \equiv \zeta(\eta, v) \in G\left(n, k^{\prime}\right) .
$$

The Radon transform of a function $f$ on $G(n, k)$ is a function $R f$ on $G\left(n, k^{\prime}\right)$ defined by

$$
(R f)(\zeta)=\int_{\tau \subset \zeta} f(\tau) d_{\zeta} \tau
$$

In terms of $(2.5)$ it means

$$
(R f)(\eta, v)=\int_{\xi \subset \eta} d_{\eta} \xi \int_{\xi^{\perp} \cap \eta} f(\xi, v+x) d x,
$$

where $d_{\eta} \xi$ denotes the canonical probability measure on the Grassmannian $G_{k}(\eta)$ of all $k$-dimensional linear subspaces of $\eta$. The right-hand side of (2.7) gives precise meaning to the integral $\int_{\tau \subset \zeta} f(\tau) d_{\zeta} \tau$ denoting integration over all $k$-planes $\tau$ in the $k^{\prime}$-plane $\zeta$. Assuming $g \in S O(n)$ to be a rotation satisfying

$$
g: \mathbb{R}^{k^{\prime}} \rightarrow \eta, \quad g: e_{k^{\prime}+1} \rightarrow v /|v|,
$$

and setting $f_{g}(\tau)=f(g \tau)$, one can write $(2.6)$ as

$$
(R f)(\eta, v)=\int_{G_{k^{\prime}, k}} d \sigma \int_{\sigma^{\perp} \cap \mathbb{R}^{k^{\prime}}} f_{g}\left(\sigma,|v| e_{k^{\prime}+1}+y\right) d y .
$$

The dual Radon transform of a function $\varphi(\zeta) \equiv \varphi(\eta, v)$ on $G\left(n, k^{\prime}\right)$ is a function $\left(R^{*} \varphi\right)(\tau) \equiv\left(R^{*} \varphi\right)(\xi, u)$ on $G_{n, k}$ defined by

$$
\begin{aligned}
\left(R^{*} \varphi\right)(\tau) & =\int_{\zeta \supset \tau} \varphi(\zeta) d_{\tau} \zeta \equiv \int_{\eta \supset \xi} \varphi(\eta+u) d_{\xi} \eta \\
& =\int_{\eta \supset \xi} \varphi\left(\eta, \operatorname{Pr}_{\eta^{\perp}} u\right) d_{\xi} \eta .
\end{aligned}
$$

Here $\operatorname{Pr}_{\eta^{\perp}} u$ is the orthogonal projection of $u\left(\in \xi^{\perp}\right)$ onto $\eta^{\perp}\left(\subset \xi^{\perp}\right)$, $d_{\xi} \eta$ is the relevant probability measure. In order to give (2.9) precise meaning, we choose a rotation $g_{\xi} \in S O(n)$ so that $g_{\xi} \mathbb{R}^{k}=\xi$, and let $K_{0} \subset S O(n)$ be the isotropy subgroup at $\mathbb{R}^{k} \in G_{n, k}$. Then (2.9) means

$$
\left(R^{*} \varphi\right)(\tau) \equiv\left(R^{*} \varphi\right)(\xi, u)=\int_{K_{0}} \varphi\left(g_{\xi} \rho \mathbb{R}^{k^{\prime}}+u\right) d \rho .
$$


Lemma 2.1. [10, Lemma 2.1] The equality

$$
\int_{G\left(n, k^{\prime}\right)}(R f)(\zeta) \varphi(\zeta) d \zeta=\int_{G(n, k)} f(\tau)\left(R^{*} \varphi\right)(\tau) d \tau
$$

holds provided that the integral in either side is finite when $f$ and $\varphi$ are replaced by $|f|$ and $|\varphi|$, respectively.

\section{Lemma 2.2.}

(i) If $f \in L^{p}(G(n, k)), 1 \leq p<(n-k) /\left(k^{\prime}-k\right)$, then $(R f)(\zeta)$ is finite for almost all $\zeta \in G\left(n, k^{\prime}\right)$. If $f \in C_{\mu}(G(n, k)), \mu>k^{\prime}-k$, then $(R f)(\zeta)$ is finite for all $\zeta \in G\left(n, k^{\prime}\right)$. The conditions $p<(n-k) /\left(k^{\prime}-k\right)$ and $\mu>k^{\prime}-k$ are sharp.

(ii) The dual transform $\left(R^{*} \varphi\right)(\tau)$ is finite a.e. on $G(n, k)$ for every locally integrable function $\varphi$ on $G\left(n, k^{\prime}\right)$ and represents a locally integrable function on $G(n, k)$.

The statement (i) is proved in [10, Corollary 2.6]. The statement (ii) follows from the equality

$$
\int_{|\tau|<a}\left(R^{*} \varphi\right)(\tau) d \tau=\mathrm{const} \int_{|\zeta|<a} \varphi(\zeta)\left(a^{2}-|\zeta|^{2}\right)^{\left(k^{\prime}-k\right) / 2} d \zeta
$$

which is a particular case of the formula (2.19) from [10].

We will also work with weighted spaces (2.1).

Lemma 2.3. [10, Proposition 1.2] For $\lambda=n-k^{\prime}$, the Radon transform (2.6) is a linear bounded operator from $L_{\lambda}^{1}(G(n, k))$ to $L_{\lambda+\delta}^{1}\left(G\left(n, k^{\prime}\right)\right)$, $\forall \delta>0$. The exponent $\lambda=n-k^{\prime}$ is best possible.

Lemma 2.4. [10, Propositions 1.3, 1.4]

(i) For $f \in L^{p}(G(n, k)), 1 \leq p<(n-k) /\left(k^{\prime}-k\right)$ or $f \in L_{n-k^{\prime}}^{1}(G(n, k))$, the Radon transform $R f$ is injective if and only if $k+k^{\prime} \leq n-1$.

(ii) For $\varphi \in L_{k+1}^{1}\left(G\left(n, k^{\prime}\right)\right)$, the dual Radon transform $R^{*} \varphi$ is injective if and only if $k+k^{\prime} \geq n-1$.

2.3. Fractional Integrals and Derivatives on the Half-Line. More information about fractional integrals in this section can be found in $[14,15]$. Let $f$ be a function on $\mathbb{R}_{+}=(0, \infty)$. For $\alpha>0$ and $t>0$, we consider two types of Riemann-Liouville fractional integrals (left-sided and right-sided) defined by

$$
\left(I_{+}^{\alpha} f\right)(t)=\frac{1}{\Gamma(\alpha)} \int_{0}^{t} \frac{f(r) d r}{(t-r)^{1-\alpha}}, \quad\left(I_{-}^{\alpha} f\right)(t)=\frac{1}{\Gamma(\alpha)} \int_{t}^{\infty} \frac{f(r) d r}{(r-t)^{1-\alpha}} .
$$


Fractional derivatives $\mathcal{D}_{ \pm}^{\alpha} \varphi$ of order $\alpha>0$ are defined as left inverses of the corresponding fractional integrals, so that

$$
\mathcal{D}_{ \pm}^{\alpha} I_{ \pm}^{\alpha} f=f .
$$

The operators $\mathcal{D}_{ \pm}^{\alpha}$ may have different analytic forms depending on the class of functions. For example, if $\alpha=m+\alpha_{0}, m=[\alpha], 0 \leq \alpha_{0}<1$, then

$$
\mathcal{D}_{ \pm}^{\alpha} \varphi=( \pm d / d t)^{m+1} I_{ \pm}^{1-\alpha_{0}} \varphi
$$

The existence of the fractional derivative and the equality (2.14) must be justified at each occurrence. The expressions (2.15) are called Riemann-Liouville fractional derivatives of $\varphi$.

We shall also work with the so-called modified Erdélyi-Kober fractional integrals having the form

$$
\begin{aligned}
& \left(I_{+, 2}^{\alpha} f\right)(t)=\frac{2}{\Gamma(\alpha)} \int_{0}^{t}\left(t^{2}-r^{2}\right)^{\alpha-1} f(r) r d r \quad \text { (left-sided) } \\
& \left(I_{-, 2}^{\alpha} f\right)(t)=\frac{2}{\Gamma(\alpha)} \int_{t}^{\infty}\left(r^{2}-t^{2}\right)^{\alpha-1} f(r) r d r \quad \text { (right-sided) }
\end{aligned}
$$

where $t>0$. Below we review basic facts from [15, Subsection 2.6.2] related to the existence of these integrals and the corresponding inversion formulas.

\section{Lemma 2.5.}

(i) The integral $\left(I_{+, 2}^{\alpha} f\right)(t)$ is absolutely convergent for almost all $t>0$ whenever $r \rightarrow r f(r)$ is a locally integrable function on $\mathbb{R}_{+}$.

(ii) Let $a>0$. If

$$
\int_{a}^{\infty}|f(r)| r^{2 \alpha-1} d r<\infty
$$

then $\left(I_{-, 2}^{\alpha} f\right)(t)$ is finite for almost all $t>a$. If $f$ is non-negative, locally integrable on $[a, \infty)$, and (2.18) fails, then $\left(I_{-, 2}^{\alpha} f\right)(t)=\infty$ for every $t \geq a$.

Fractional derivatives of the Erdélyi-Kober type are defined as the left inverses $\mathcal{D}_{ \pm, 2}^{\alpha}=\left(I_{ \pm, 2}^{\alpha}\right)^{-1}$. We have

$$
\mathcal{D}_{ \pm, 2}^{\alpha} \varphi=\Lambda^{-1} \mathcal{D}_{ \pm}^{\alpha} \Lambda \varphi, \quad(\Lambda f)(t)=f(\sqrt{t}),
$$

where the Riemann-Liouville derivatives $\mathcal{D}_{ \pm}^{\alpha}$ can be chosen in different forms, depending on our needs. For example, if $\alpha=m+\alpha_{0}, m=$ 
$[\alpha], 0 \leq \alpha_{0}<1$, then, formally, (2.15) yields

$$
\mathcal{D}_{ \pm, 2}^{\alpha} \varphi=( \pm D)^{m+1} I_{ \pm, 2}^{1-\alpha_{0}} \varphi, \quad D=\frac{1}{2 t} \frac{d}{d t} .
$$

This formula is well-justified in the " + " case when $\varphi=I_{+, 2}^{\alpha} f$ with $r f(r)$ being locally integrable on $\mathbb{R}_{+}$; cf. Lemma 2.5(i).

Inversion of the operator $I_{-, 2}^{\alpha}$ deserves special consideration because the analytic expression of $\mathcal{D}_{-, 2}^{\alpha}$ essentially depends on the behavior of functions at infinity.

Theorem 2.6. Let $\varphi=I_{-, 2}^{\alpha} f$, where $f$ satisfies (2.18) for every $a>0$. Then $f(t)=\left(\mathcal{D}_{-, 2}^{\alpha} \varphi\right)(t)$ for almost all $t \in \mathbb{R}_{+}$and $\mathcal{D}_{-, 2}^{\alpha} \varphi$ has one of the following forms.

(i) If $\alpha=m$ is an integer, then

$$
\mathcal{D}_{-, 2}^{\alpha} \varphi=(-D)^{m} \varphi, \quad D=\frac{1}{2 t} \frac{d}{d t} .
$$

(ii) If $\alpha=m+\alpha_{0}, m=[\alpha], 0<\alpha_{0}<1$, then

$$
\mathcal{D}_{-, 2}^{\alpha} \varphi=t^{2(1-\alpha+m)}(-D)^{m+1} t^{2 \alpha} \psi, \quad \psi=I_{-, 2}^{1-\alpha+m} t^{-2 m-2} \varphi .
$$

In particular, for $\alpha=k / 2, k$ odd,

$$
\mathcal{D}_{-, 2}^{k / 2} \varphi=t(-D)^{(k+1) / 2} t^{k} I_{-, 2}^{1 / 2} t^{-k-1} \varphi .
$$

Alternatively,

$$
\mathcal{D}_{-, 2}^{\alpha} \varphi=2^{-2 \alpha} \mathcal{D}_{-}^{2 \alpha} t I_{-, 2}^{\alpha} t^{-2 \alpha-1} \varphi,
$$

where $\mathcal{D}_{-}^{2 \alpha}$ denotes the Riemann-Liouville fractional derivative of order $2 \alpha$ (cf. (2.15)).

If, moreover, $\int_{a}^{\infty}|f(t)| t^{2 m+1} d t<\infty$ for all $a>0$, then

$$
\mathcal{D}_{-, 2}^{\alpha} \varphi=(-D)^{m+1} I_{-, 2}^{1-\alpha+m} \varphi \text {. }
$$

Many other inversion formulas for fractional integrals can be found in $[15$, Section 2.4].

2.4. Radon-John Transforms. Given an integer $0<d<n$, the Radon-John $d$-plane transform of a function $f$ on $\mathbb{R}^{n}$ is a function $R_{d} f$ on $G(n, d)$ defined by the integral

$$
\left(R_{d} f\right)(\tau)=\int_{\tau} f(x) d_{\tau} x,
$$

where $d_{\tau} x$ stands for the Euclidean volume element of the plane $\tau$. The existence of this integral depends on the class of functions $f$. If $f \in C_{\mu}\left(\mathbb{R}^{n}\right), \mu>d$, then $\left(R_{d} f\right)(\tau)$ is finite for all $\tau \in G(n, d)$. If $f \in L^{p}\left(\mathbb{R}^{n}\right), 1 \leq p<n / d$, then $\left(R_{d} f\right)(\tau)$ is finite a.e. on $G(n, d)$. 
Both restriction $\mu>d$ and $1 \leq p<n / d$ are sharp. We also have the following statement which is a reformulation of Theorem 3.2 from [14].

Lemma 2.7. If

$$
\int_{\mathbb{R}^{n}} \frac{|f(x)|}{(1+|x|)^{n-d}} d x<\infty,
$$

then $\left(R_{d} f\right)(\tau)$ is finite for almost all $\tau \in G(n, d)$. If $f$ is nonnegative, radial, and (2.27) fails, then $\left(R_{d} f\right)(\tau) \equiv \infty$.

For $x \in \mathbb{R}^{n}$ and $\tau \in G(n, d)$, we denote by

$$
r=|x|=\operatorname{dist}(o, x), \quad s=|\tau|=\operatorname{dist}(o, \tau)
$$

the corresponding distances from the origin.

Lemma 2.8. (cf. [11, Lemma 2.1]) If $f$ is a radial function on $\mathbb{R}^{n}$ satisfying (2.27), then $R_{d} f$ is a radial function on $G(n, d)$. Moreover, if $f(x) \equiv f_{0}(r)$ and $\left(R_{d} f\right)(\tau) \equiv F_{0}(s)$, then

$$
\begin{aligned}
F_{0}(s) & =\sigma_{d-1} \int_{s}^{\infty} f_{0}(r)\left(r^{2}-s^{2}\right)^{d / 2-1} r d r \\
& =\pi^{d / 2}\left(I_{-, 2}^{d / 2} f_{0}\right)(s) .
\end{aligned}
$$

A variety of inversion formulas for the $d$-plane transform are known; see, e.g., $[1,8,11,12,14]$ and references therein. For example, the following theorems were proved in [14].

Theorem 2.9. A function $f \in C_{\mu}\left(\mathbb{R}^{n}\right), \mu>d$, can be recovered from $\varphi=R_{d} f$ by the formula

$$
\begin{gathered}
f(x)=\left(R_{d}^{-1} \varphi\right)(x)=\lim _{t \rightarrow 0} \pi^{-d / 2}\left(\mathcal{D}_{-, 2}^{d / 2} F_{x}\right)(t), \\
F_{x}(t)=\int_{S O(n)} \varphi\left(\gamma \mathbb{R}^{d}+x+t \gamma e_{n}\right) d \gamma,
\end{gathered}
$$

where the limit is uniform on $\mathbb{R}^{n}$ and the Erdélyi-Kober differential operator $\mathcal{D}_{-, 2}^{d / 2}$ can be computed as follows.

(i) If $d$ is even, then

$$
\mathcal{D}_{-, 2}^{d / 2} F_{x}=(-D)^{d / 2} F_{x}, \quad D=\frac{1}{2 t} \frac{d}{d t} .
$$

(ii) For any $1 \leq d \leq n-1$,

$$
\mathcal{D}_{-, 2}^{d / 2} F_{x}=t^{2-d+2 m}(-D)^{m+1} t^{d} \psi, \quad \psi=I_{-, 2}^{1-d / 2+m} t^{-2 m-2} F_{x},
$$


where $m=[d / 2]$. Alternatively,

$$
\mathcal{D}_{-, 2}^{d / 2} F_{x}=2^{-d}\left(-\frac{d}{d t}\right)^{d} t I_{-, 2}^{d / 2} t^{-d-1} F_{x} .
$$

Under the stronger assumption $\mu>2+2[d / 2](>d), \mathcal{D}_{-, 2}^{d / 2}$ can also be computed as

$$
\mathcal{D}_{-, 2}^{d / 2} F_{x}=(-D)^{m+1} I_{-, 2}^{1-\alpha+m} F_{x} .
$$

Note that powers of $t$ in these formulas stand for the corresponding multiplication operators.

The next theorem contains similar results for $L^{p}$-functions.

Theorem 2.10. A function $f \in L^{p}\left(\mathbb{R}^{n}\right), 1 \leq p<n / d$, can be recovered from $\varphi=R_{d} f$ at almost every $x \in \mathbb{R}^{n}$ by the formula

$$
f(x)=\left(R_{d}^{-1} \varphi\right)(x)=\lim _{t \rightarrow 0} \pi^{-d / 2}\left(\mathcal{D}_{-, 2}^{d / 2} F_{x}\right)(t),
$$

where the limit is understood in the $L^{p}$-norm. Here $F_{x}$ is defined by (2.32) and $\mathcal{D}_{-, 2}^{d / 2} F_{x}$ is computed as in Theorem 2.9, where (2.36) is applicable under the stronger assumption $1 \leq p<n /(2+2[d / 2])$.

\subsection{The Funk-Radon Transforms on Compact Grassmanni-}

ans. Let $G_{n, k}$ and $G_{n, k^{\prime}}$ be a pair of compact Grassmann manifolds of linear subspaces of $\mathbb{R}^{n}, 0<k<k^{\prime}<n$. For a function $\Phi$ on $G_{n, k}$, we consider the Funk-Radon transform ${ }^{1}$

$$
\left(\mathcal{F}_{(n)} \Phi\right)(\eta)=\int_{\xi \subset \eta} \Phi(\xi) d_{\eta} \xi, \quad \eta \in G_{n, k^{\prime}},
$$

that integrates $\Phi$ over the set $G_{k}(\eta)$ of all $k$-dimensional subspaces of $\eta$ with respect to the corresponding probability measure on $G_{k}(\eta)$. If $g_{\eta}$ is a rotation satisfying $g_{\eta} \mathbb{R}^{k^{\prime}}=\eta$, then

$$
\left(\mathcal{F}_{(n)} \Phi\right)(\eta)=\int_{G_{k^{\prime}, k}} \Phi\left(g_{\eta} \xi\right) d \xi
$$

The dual Funk-Radon transform $\mathcal{F}_{(n)}^{*} \Psi$ of a function $\Psi$ on $G_{n, k^{\prime}}$ integrates $\Psi$ over the set of all $k^{\prime}$-dimensional subspaces $\eta$ containing the $k$-dimensional subspace $\xi$, namely,

$$
\left(\mathcal{F}_{(n)}^{*} \Psi\right)(\xi)=\int_{\eta \supset \xi} \Psi(\eta) d_{\xi} \eta, \quad \xi \in G_{n, k} .
$$

\footnotetext{
${ }^{1}$ The subscript " $(n)$ " below is intentional. In Section 3 we will be dealing with the similar transform $\mathcal{F}_{(\ell)}, \ell<n$, associated with Grassmannians $G_{\ell, k}$ and $G_{\ell, k^{\prime}}$.
} 
To give this integral precise meaning, we denote by $g_{\xi}$ a rotation satisfying $g_{\xi} \mathbb{R}^{k}=\xi$ and let $K_{0} \subset S O(n)$ be the isotropy subgroup at $\mathbb{R}^{k} \in G_{n, k}$. Then (2.40) is understood as follows:

$$
\left(\mathcal{F}_{(n)}^{*} \Psi\right)(\xi)=\int_{K_{0}} \Psi\left(g_{\xi} \rho \mathbb{R}^{k^{\prime}}\right) d \rho
$$

\section{Lemma 2.11.}

(i) The Funk-Radon transform $\mathcal{F}_{(n)}$ and its dual $\mathcal{F}_{(n)}^{*}$ are linear bounded operators from $L^{1}\left(G_{n, k}\right)$ to $L^{1}\left(G_{n, k^{\prime}}\right)$, and from $L^{1}\left(G_{n, k^{\prime}}\right)$ to $L^{1}\left(G_{n, k}\right)$, respectively.

(ii) The duality relation

$$
\int_{G_{n, k^{\prime}}}\left(\mathcal{F}_{(n)} \Phi\right)(\eta) \Psi(\eta) d \eta=\int_{G_{n, k}} \Phi(\xi)\left(\mathcal{F}_{(n)}^{*} \Psi\right)(\xi) d \xi
$$

holds provided that the integral in either side of this equality is finite when $\Phi$ and $\Psi$ are replaced by $|\Phi|$ and $|\Psi|$, respectively. In particular, for $\Phi \in L^{1}\left(G_{n, k}\right)$,

$$
\int_{G_{n, k^{\prime}}}\left(\mathcal{F}_{(n)} \Phi\right)(\eta) d \eta=\int_{G_{n, k}} \Phi(\xi) d \xi
$$

We review some facts from [6] in our notation. Let $\mathcal{P}_{k}$ be the cone of positive definite symmetric $k \times k$ matrices $r=\left(r_{i, j}\right)$. The Siegel gamma function associated to $\mathcal{P}_{k}$ is defined by

$$
\begin{gathered}
\Gamma_{k}(\alpha)=\int_{\mathcal{P}_{k}} e^{-\operatorname{tr}(r)} \operatorname{det}(r)^{\alpha-d} d r, \\
d=(k+1) / 2, \quad d r=\prod_{i \leq j} d r_{i, j}, \quad \operatorname{tr}(r)=\text { trace of } r .
\end{gathered}
$$

This integral converges for all $\operatorname{Re} \alpha>d-1$ and represents a product of usual gamma functions:

$$
\Gamma_{k}(\alpha)=\pi^{k(k-1) / 4} \Gamma(\alpha) \Gamma\left(\alpha-\frac{1}{2}\right) \ldots \Gamma\left(\alpha-\frac{k-1}{2}\right) .
$$

The Gårding-Gindikin fractional integral of a function $f$ on $\mathcal{P}_{k}$ is defined by

$$
\left(I_{+}^{\alpha} f\right)(r)=\frac{1}{\Gamma_{k}(\alpha)} \int_{0}^{r} f(s) \operatorname{det}(r-s)^{\alpha-d} d s, \quad \text { Re } \alpha>d-1
$$


where $\int_{0}^{r}$ denotes integration over the set $\left\{s: s \in \mathcal{P}_{k}, r-s \in \mathcal{P}_{k}\right\}$. We define a differential operator (in the $r$-variable):

$$
D_{+}=\operatorname{det}\left(\eta_{i, j} \frac{\partial}{\partial r_{i, j}}\right), \quad \eta_{i, j}= \begin{cases}1 & \text { if } i=j, \\ 1 / 2 & \text { if } i \neq j\end{cases}
$$

so that

$$
D_{+}^{m} I_{+}^{\alpha} f=I_{+}^{\alpha-m} f, \quad m \in \mathbb{N}, \quad \text { Re } \alpha>m+d-1 .
$$

This equality holds pointwise if $f$ is good enough and is understood in the sense of distributions, otherwise.

Let $\xi \in G_{n, k}, \eta \in G_{n, k^{\prime}}, 1 \leq k<k^{\prime} \leq n-1$. Given a function $\Psi$ on $G_{n, k^{\prime}}$, we introduce the mean value operator

$$
\left(M_{r}^{*} \Psi\right)(\xi)=\int_{O(k)} d u \int_{\left\{\eta: \operatorname{Cos}^{2}(\eta, y)=u^{T} r u\right\}} \Psi(\eta) d m_{\xi}(\eta), \quad r \in \mathcal{P}_{k},
$$

where $\operatorname{Cos}^{2}(\eta, y) \stackrel{\text { def }}{=} y^{T} \operatorname{Pr}_{\eta} y, y$ is a matrix whose columns form an orthonormal basis of $\xi$. Changing variables $y \rightarrow y \gamma, u \rightarrow u \gamma$ with $\gamma \in O(k)$, one can readily see that the right-hand side of (2.48) is independent of the choice of the orthonormal basis $y$ of $\xi$. Detailed explanation of the definition of the matrix-valued Cos-function are given in $[6$, Section 3] and [18, p. 156].

Theorem 2.12. [6, Theorem 1.2] Let $\Phi \in L^{1}\left(G_{n, k}\right)$. Suppose that

$$
\Psi(\eta)=\left(\mathcal{F}_{(n)} \Phi\right)(\eta), \quad \eta \in G_{n, k^{\prime}}, \quad 1 \leq k<k^{\prime} \leq n-1,
$$

and denote

$$
\alpha=\frac{k^{\prime}-k}{2}, \quad \tilde{\Psi}_{\xi}(r)=\operatorname{det}(r)^{\alpha-1 / 2}\left(M_{r}^{*} \Psi\right)(\xi), \quad \xi \in G_{n, k} .
$$

The operator $\mathcal{F}_{(n)}$ is injective if and only if

$$
k+k^{\prime} \leq n \text {. }
$$

Under this condition, the function $\Phi$ can be recovered by the formula $\Phi=\mathcal{F}_{(n)}^{-1} \Psi$, where

$$
\left(\mathcal{F}_{(n)}^{-1} \Psi\right)(\xi)=c \lim _{r \rightarrow I_{k}}^{\left(L^{1}\right)}\left(D_{+}^{m} I_{+}^{m-\alpha} \tilde{\Psi}_{\xi}\right)(r), \quad c=\frac{\Gamma_{k}(k / 2)}{\Gamma_{k}\left(k^{\prime} / 2\right)} .
$$

Here $m$ is an arbitrary integer greater than $\left(k^{\prime}-1\right) / 2, I_{k}$, is the identity $k \times k$ matrix, and the differentiation is understood in the sense of distributions. In particular, for $k^{\prime}-k=2 \ell, \ell \in \mathbb{N}$, we have

$$
\left(\mathcal{F}_{(n)}^{-1} \Psi\right)(\xi)=c \lim _{r \rightarrow I_{k}}^{\left(L^{1}\right)}\left(D_{+}^{\ell} \tilde{\Psi}_{\xi}\right)(r)
$$


If $\Phi$ is a continuous function on $G_{n, k}$, then the limit in (2.50) and (2.51) can be understood in the sup-norm.

Remark 2.13. Since the Funk-Radon transform and its dual can be expressed one through another, Theorem 2.12 implies the corresponding inversion result for $\mathcal{F}_{(n)}^{*}$. In general, every function $\psi$ on $G_{n, \ell}$ can be regarded as a function on $G_{n, n-\ell}$ if we set $(T \psi)\left(h^{\perp}\right)=\psi(h), h \in G_{n, \ell}$. One can readily show (cf. [10, Lemma 4.3]) that

$$
\int_{G_{n, n-\ell}}(T \psi)\left(h^{\perp}\right) d h^{\perp}=\int_{G_{n, \ell}} \psi(h) d h,
$$

where $d h$ and $d h^{\perp}$ are the corresponding probability measures. Thus $\mathcal{F}_{(n)}^{*}=T \tilde{\mathcal{F}}_{(n)} T$, where $\tilde{\mathcal{F}}_{(n)}$ is the Funk-Radon transform for a pair of Grassmannians $G_{n, n-k^{\prime}}$ and $G_{n, n-k}$. It follows that

$$
\left(\mathcal{F}_{(n)}^{*}\right)^{-1}=T\left(\tilde{\mathcal{F}}_{(n)}\right)^{-1} T
$$

\section{Inversion of the Radon Transform for a Pair of Affine Grassmannians}

The main idea is to reduce the problem to known inversion formulas in compact and non-compact settings by making use of a certain factorization procedure. Subsection 3.1 is devoted to Radon transforms of the so-called quasi-radial functions satisfying certain symmetry. The general case is considered in Subsection 3.2.

\subsection{Radon Transforms of Quasi-Radial Functions.}

Definition 3.1. A function $f: G(n, k) \rightarrow \mathbb{C}$ is called quasi-radial if $f(\xi, \cdot)$ is a radial function on $\xi^{\perp}$ for every $\xi \in G_{n, k}$, in other words, if $f(\xi, u)=f_{0}(\xi,|u|)$ for some function $f_{0}$ on $G_{n, k} \times \mathbb{R}_{+}$.

Lemma 3.2. If $f(\xi, u) \equiv f_{0}(\xi,|u|)$ is a quasi-radial function on $G(n, k)$ satisfying

$$
\int_{G_{n, k}} d \xi \int_{a}^{\infty}\left|f_{0}(\xi, r)\right| r^{k^{\prime}-k-1} d r<\infty, \quad \forall a>0,
$$

then the Radon transform $\varphi=R f$, where

$$
(R f)(\eta, v) \equiv \int_{\xi \subset \eta} d_{\eta} \xi \int_{\xi^{\perp} \cap \eta} f(\xi, v+x) d x
$$


is quasi-radial too. Moreover, if $\varphi(\eta, v)=\varphi_{0}(\eta,|v|)$, then

$$
\varphi_{0}(\eta, s)=\int_{\xi \subset \eta} g_{\xi}(s) d_{\eta} \xi
$$

where

$$
g_{\xi}(s)=\sigma_{k^{\prime}-k-1} \int_{s}^{\infty} f_{0}(\xi, r)\left(r^{2}-s^{2}\right)^{\left(k^{\prime}-k\right) / 2-1} r d r .
$$

Proof. For fixed $\eta$ and $\xi$, the inner integral in (3.2) is the Radon-John transform of the function $f(\xi, \cdot): \xi^{\perp} \rightarrow \mathbb{C}$ over the $\left(k^{\prime}-k\right)$-dimensional plane $\left(\xi^{\perp} \cap \eta\right)+v$ in $\xi^{\perp}\left(\sim \mathbb{R}^{n-k}\right)$. If $f(\xi, \cdot)$ is radial on $\xi^{\perp}$, the result follows from Lemmas 2.8 and 2.5.

The right-hand side of (3.3) is a constant multiple of the tensor product of the Funk-Radon transform (2.38) and the Erdélyi-Kobertype operator (2.17). Combining Theorems 2.12 and 2.6, we obtain the following inversion result.

Theorem 3.3. Let $\varphi=R f, 1 \leq k<k^{\prime} \leq n-1, f(\xi, u)=f_{0}(\xi,|u|)$, where $f_{0}$ satisfies (3.1). Suppose that $\varphi \equiv \varphi_{0}(\eta, s) \equiv \varphi_{s}(\eta)$; cf. Lemma 3.2. Then

$$
f_{0}(\xi, r)=\pi^{-\alpha}\left(D_{-, 2}^{\alpha} h_{\xi}\right)(r), \quad h_{\xi}(s)=\left(\mathcal{F}_{(n)}^{-1} \varphi_{s}\right)(\xi), \quad \alpha=\frac{k^{\prime}-k}{2},
$$

where $\mathcal{F}_{(n)}^{-1}$ is defined by (2.50),

$$
\left(D_{-, 2}^{\alpha} h_{\xi}\right)(r)=(-D)^{\alpha} h_{\xi}, \quad D=\frac{1}{2 r} \frac{d}{d r},
$$

if $\alpha$ is an integer, and

$$
\left(D_{-, 2}^{\alpha} h_{\xi}\right)(r)=r(-D)^{\alpha+1 / 2} r^{2 \alpha} I_{-, 2}^{1 / 2} r^{-2 \alpha-1} h_{\xi},
$$

otherwise $e^{2}$.

Proof. To prove this theorem, it remains to note that both Theorems 2.12 and 2.6 are applicable owing to (3.1). For example, the assumptions of Theorems 2.12 are satisfied because $g(\cdot, s) \in L^{1}\left(G_{n, k}\right)$. Indeed,

$$
\int_{G_{n, k}}|g(\xi, s)| d \xi \leq \mathrm{const} \int_{s}^{\infty}\left(r^{2}-s^{2}\right)^{\alpha-1} r d r \int_{G_{n, k}}\left|f_{0}(\xi, r)\right| d \xi<\infty
$$

for almost all $s>0$ according to Lemma 2.5(ii) and (3.1).

\footnotetext{
${ }^{2} \mathrm{As}$ in Theorem 2.6, powers of $r$ stand for the corresponding multiplication operators.
} 
3.2. The General Case. Our approach is inspired by Gonzalez and Kakehi [3, pp. 255, 258] who applied the Fourier transform to $(R f)(\eta, v)$ in the $v$-variable. However, the use of the Fourier transform leads to inevitable restrictions on the class of functions. As we shall see below, these restrictions can be essentially weakened if the Fourier transform is replaced by the suitable Radon-John transform, the $L^{p}$ theory of which is well-developed; see, e.g., [11, 14].

Let $\varkappa$ be an integer satisfying

$$
k \leq \varkappa<n-k^{\prime}
$$

(the role of $\varkappa$ will become clear from the reasoning below) and consider an auxiliary function

$$
g_{h, \alpha}(\xi) \equiv g(h, \xi, \alpha)=\int_{\xi^{\perp} \cap h} f(\xi, y+\alpha) d y
$$

on the set of triples

$$
\Omega=\left\{(h, \xi, \alpha): h \in G_{n, k^{\prime}+\varkappa}, \xi \in G_{k}(h), \alpha \in h^{\perp}\right\} .
$$

We recall that $f$ is a function on $G_{n, k}, 0<k<k^{\prime}<n$, and note that $\operatorname{dim}\left(\xi^{\perp} \cap h\right)>0$ because $\operatorname{dim}\left(\xi^{\perp}\right)+\operatorname{dim}(h)=n-k+k^{\prime}+\varkappa>n$.

Our first step is to reconstruct $g(h, \xi, \alpha)$ on $\Omega$ from $(R f)(\eta, v)$. The second step is to reconstruct $f(\xi, u)$ on $G(n, k)$ from $g(h, \xi, \alpha)$.

STEP 1. We replace the target space $G\left(n, k^{\prime}\right)$ by the "bigger" Grassmannian $G\left(n, k^{\prime}+\varkappa\right)$ of $\left(k^{\prime}+\varkappa\right)$-dimensional planes $\tilde{\zeta} \equiv \tilde{\zeta}(h, \alpha)$ in $\mathbb{R}^{n}$ and consider the corresponding Radon transform

$$
\begin{aligned}
(\tilde{R} f)(\tilde{\zeta}) & \equiv(\tilde{R} f)(h, \alpha)=\int_{\xi \subset h} d_{h} \xi \int_{\xi^{\perp} \cap h} f(\xi, y+\alpha) d y \\
& =\int_{G_{k}(h)} g_{h, \alpha}(\xi) d_{h} \xi, \quad \tilde{\zeta} \in G\left(n, k^{\prime}+\varkappa\right) .
\end{aligned}
$$

\section{Lemma 3.4.}

(i) If

$$
f \in L^{p}(G(n, k)), \quad 1 \leq p<\frac{n-k}{k^{\prime}-k+\varkappa},
$$

then $(\tilde{R}|f|)(\tilde{\zeta})$ is finite for almost all $\tilde{\zeta} \in G\left(n, k^{\prime}+\varkappa\right)$. For all such $\tilde{\zeta} \equiv \tilde{\zeta}(h, \alpha)$, we have $g_{h, \alpha} \in L^{1}\left(G_{k}(h)\right)$ and for almost all $\eta \in G_{k^{\prime}}(h)$,

$$
\int_{\xi \subset \eta} g_{h, \alpha}(\xi) d_{\eta} \xi=\int_{\pi}(R f)(\eta, v) d_{\pi} v .
$$


Here $\pi=\left(\eta^{\perp} \cap h\right)+\alpha$ is a $\varkappa$-dimensional affine plane in $\eta^{\perp}$ parallel to $h$ and $R f$ is the Radon transform (3.2).

(ii) If $f \in C_{\mu}(G(n, k)), \mu>k^{\prime}-k+\varkappa$, then $(\tilde{R}|f|)(\tilde{\zeta})$ is finite for all $\tilde{\zeta} \in G\left(n, k^{\prime}+\varkappa\right), g_{h, \alpha}$ is a continuous function on $G_{k}(h)$, and (3.13) holds for all $\eta \in G_{k^{\prime}}(h)$.

Proof. (i) The first statement follows from Lemma 2.2(i), in which $k^{\prime}$ should be replaced by $k^{\prime}+\varkappa$. This also gives $g_{h, \alpha} \in L^{1}\left(G_{k}(h)\right)$ for almost all $\tilde{\zeta} \equiv \tilde{\zeta}(h, \alpha) \in G\left(n, k^{\prime}+\varkappa\right)$. To prove (3.13), we formally have

$$
\int_{\pi}(R f)(\eta, v) d_{\pi} v=\int_{\xi \subset \eta} d_{\eta} \xi \int_{\xi^{\perp} \cap \eta} d x \int_{\eta^{\perp} \cap h} f\left(\xi, x+v_{1}+\alpha\right) d v_{1} .
$$

Keeping in mind that $\xi \subset \eta$ and therefore $\eta^{\perp} \subset \xi^{\perp}$, we observe that every vector $V$ in $\xi^{\perp}$ can be decomposed as

$$
V=\operatorname{Pr}_{h^{\perp}} V+\operatorname{Pr}_{\xi^{\perp} \cap \eta} V+\operatorname{Pr}_{\eta^{\perp} \cap h} V .
$$

Hence the right-hand side of (3.14) can be written as

$$
\int_{\xi \subset \eta} d_{\eta} \xi \int_{\xi^{\perp} \cap h} f(\xi, y+\alpha) d y=\int_{\xi \subset \eta} g_{h, \alpha}(\xi) d_{\eta} \xi,
$$

as desired. To make this reasoning rigorous, we observe that for the integral (3.11) with a non-negative function $f$ satisfying (3.12) we have

$$
(\tilde{R} f)(h, \alpha)=\int_{\eta \subset h}\left[\int_{\xi \subset \eta} d_{\eta} \xi \int_{\xi^{\perp} \cap h} f(\xi, y+\alpha) d y\right] d_{h} \eta<\infty
$$

for almost all $\tilde{\zeta}(h, \alpha) \in G\left(n, k^{\prime}+\varkappa\right)$; cf. (2.43). It follows that the expression in square brackets is finite for almost all $\eta \in G_{k^{\prime}}(h)$. But this expression is exactly the left-hand side of (3.15). This completes the proof for the $L^{p}$ - case.

(ii) If $f \in C_{\mu}(G(n, k))$ with $\mu>k^{\prime}-k+\varkappa$, then $(\tilde{R}|f|)(\tilde{\zeta})$ is finite for all $\tilde{\zeta} \in G\left(n, k^{\prime}+\varkappa\right)$ by Lemma 2.2 (i). Moreover, $f \in L^{p}(G(n, k))$ whenever

$$
\frac{n-k}{\mu} \leq p<\frac{n-k}{k^{\prime}-k+\varkappa}
$$

and therefore all statements in (i) remain true. The continuity of $g_{h, \alpha}$ follows immediately from its definition (3.9). The validity of (3.13) for all $\eta \in G_{k^{\prime}}(h)$ is a consequence of continuity of all functions in this equality. 
Lemma 3.4 allows us to reconstruct $g(h, \xi, \alpha)$ on $\Omega$ from $(R f)(\eta, v)$. Indeed, denote

$$
G_{h, \alpha}(\eta)=\int_{\pi}(R f)(\eta, v) d_{\pi} v, \quad \pi=\left(\eta^{\perp} \cap h\right)+\alpha .
$$

By (3.13),

$$
\int_{\xi \subset \eta} g_{h, \alpha}(\xi) d_{\eta} \xi=G_{h, \alpha}(\eta), \quad \eta \in G_{k^{\prime}}(h) .
$$

The left-hand side is the Funk-Radon transform of $g_{h, \alpha}$ for a pair of compact Grassmannians $G_{k}(h)$ and $G_{k^{\prime}}(h)$. Let

$$
\ell=k^{\prime}+\varkappa, \quad \mathbb{R}^{\ell}=\mathbb{R} e_{1} \oplus \cdots \oplus \mathbb{R} e_{\ell},
$$

and let $\mathcal{F}_{(\ell)}$ be the Funk-Radon transform for a pair of compact Grassmannians $G_{\ell, k}$ and $G_{\ell, k^{\prime}}$; cf. (2.6) for $\ell=n$. If $\gamma_{h}$ is a rotation that takes $\mathbb{R}^{\ell}$ to $h$, then $(3.18)$ can be written as

$$
\mathcal{F}_{(\ell)}\left[g_{h, \alpha} \circ \gamma_{h}\right]=G_{h, \alpha} \circ \gamma_{h} .
$$

Because $g_{h, \alpha} \in L^{1}\left(G_{k}(h)\right)$, it follows that $g_{h, \alpha} \circ \gamma_{h} \in L^{1}\left(G_{\ell, k}\right)$, and the operator $\mathcal{F}_{(\ell)}$ can be explicitly inverted by Theorem 2.12. This gives

$$
g_{h, \alpha}=\mathcal{F}_{(\ell)}^{-1}\left[G_{h, \alpha} \circ \gamma_{h}\right] \circ \gamma_{h}^{-1},
$$

where $\mathcal{F}_{(\ell)}^{-1}$ is defined by $(2.50)$ with $n$ replaced by $\ell=k^{\prime}+\varkappa$. Thus we have proved the following

Proposition 3.5. Let $0<k<k^{\prime}<n, k \leq \varkappa<n-k^{\prime}$. If $f \in$ $L^{p}(G(n, k)), 1 \leq p<(n-k) /\left(k^{\prime}-k+\varkappa\right)$, then, for almost all $\tilde{\zeta}(h, \alpha) \in$ $G\left(n, k^{\prime}+\varkappa\right)$, the function $g_{h, \alpha}$ from (3.9) can be expressed through the Radon transform $R f$ by the formula (3.19) in which $G_{h, \alpha}$ is defined by (3.17). If $f \in C_{\mu}(G(n, k)), \mu>k^{\prime}-k+\varkappa$, the above statement holds for all $\tilde{\zeta}(h, \alpha) \in G\left(n, k^{\prime}+\varkappa\right)$.

STEP 2. Our next aim is to reconstruct $f(\tau) \equiv f(\xi, u)$ on $G(n, k)$ from $g(h, \xi, \alpha)$. To this end, we fix $\xi \in G_{n, k}$ and choose a plane $\tilde{\zeta}(h, \alpha) \in G\left(n, k^{\prime}+\varkappa\right)$, so that $h \supset \xi$ and $g(h, \xi, \alpha)$ can be found by Step 1 . Every triple $(h, \xi, \alpha)$ obtained this way belongs to the set (3.10) and determines a plane $\omega$ in $\xi^{\perp}$ so that

$$
\omega=\left(h \cap \xi^{\perp}\right)+\alpha, \quad \operatorname{dim}(\omega)=k^{\prime}-k+\varkappa \stackrel{\text { def }}{=} k_{1} .
$$

Note that every $k_{1}$-plane $\omega$ in $\xi^{\perp}$ is uniquely represented in the form (3.20) with some $h \in G_{n, k^{\prime}+\varkappa}$ containing $\xi$ and some $\alpha \in h^{\perp}$. Indeed, we can write $\omega=\omega_{0}+\alpha$, where $\omega_{0} \in G_{k_{1}}\left(\xi^{\perp}\right)$ is parallel to $\omega$ and 
$\alpha \in \omega_{0}^{\perp} \cap \xi^{\perp}$. It remains to set $h=\omega_{0} \oplus \xi$ and note that $h^{\perp}=$ $\left(\omega_{0} \oplus \xi\right)^{\perp}=\omega_{0}^{\perp} \cap \xi^{\perp} \ni \alpha$.

Now we set $f_{\xi}(u) \equiv f(\xi, u)$ and write (3.9) as

$$
g(h, \xi, \alpha) \equiv \int_{\xi^{\perp} \cap h} f_{\xi}(y+\alpha) d y \stackrel{\text { def }}{=} G_{\xi}(\omega), \quad \omega=\left(h \cap \xi^{\perp}\right)+\alpha .
$$

This is the Radon-John $k_{1}$-plane transform $\left(R_{k_{1}} f_{\xi}\right)(\omega)$ of the function $f_{\xi} \equiv f(\xi, \cdot)$ defined on $\xi^{\perp}$. The operator $R_{k_{1}}$ can be explicitly inverted by making use of Theorems 2.9 and 2.10 adjusted to our case. Specifically, we replace the ambient space $\mathbb{R}^{n}$ in $(2.37)$ by $\xi^{\perp}, d$ by $k_{1}, f(x)$ by $f_{\xi}(u)$, and the average $F_{x}(t)($ cf. $(2.32))$ by

$$
F_{\xi, u}(t)=\int_{S O\left(\xi^{\perp}\right)} G_{\xi}\left(\gamma \omega_{\xi, t}+u\right) d \gamma
$$

Here $S O\left(\xi^{\perp}\right) \subset S O(n)$ is the isotropy subgroup of $\xi^{\perp}, \omega_{\xi, t}$ is an arbitrary $k_{1}$-plane in $\xi^{\perp}$ at distance $t>0$ from the origin.

The above reasoning leads to the following statement.

Proposition 3.6. Let $0<k<k^{\prime}<n, k \leq \varkappa<n-k^{\prime}$. If $f \in$ $L^{p}(G(n, k)), 1 \leq p<(n-k) /\left(k^{\prime}-k+\varkappa\right)$, then for almost all $\tau \equiv$ $\tau(\xi, u) \in G(n, k)$, the function $f(\tau) \equiv f_{\xi}(u)$ can be reconstructed from $g(h, \xi, \alpha) \equiv G_{\xi}(\omega), \omega=\left(h \cap \xi^{\perp}\right)+\alpha \in G\left(\xi^{\perp}, k_{1}\right)$, by the formula

$$
f_{\xi}(u)=\lim _{t \rightarrow 0} \pi^{-k_{1} / 2}\left(\mathcal{D}_{-, 2}^{k_{1} / 2} F_{\xi, u}\right)(t),
$$

where, for almost all $\xi \in G_{n, k}$, the limit is understood in the $L^{p}\left(\xi^{\perp}\right)$ norm. Here $F_{\xi, u}$ is defined by (3.22) and $\mathcal{D}_{-, 2}^{k_{1} / 2} F_{\xi, u}$ is computed as in Theorem 2.9. If $f \in C_{\mu}(G(n, k)), \mu>k^{\prime}-k+\varkappa$, the above statement holds for all $\xi \in G_{n, k}$ and the limit in (3.23) is understood in the supnorm on $\xi^{\perp}$.

Combining Propositions 3.5 and 3.6, we obtain the following result.

Theorem 3.7. Let $0<k<k^{\prime}<n, k \leq \varkappa<n-k^{\prime}$. Suppose that

$$
f \in C_{\mu}(G(n, k)), \quad \mu>k^{\prime}-k+\varkappa,
$$

or

$$
f \in L^{p}(G(n, k)), \quad 1 \leq p<(n-k) /\left(k^{\prime}-k+\varkappa\right) .
$$

Then $f(\tau) \equiv f_{\xi}(u)$ can be reconstructed for all or almost all $\tau \equiv$ $\tau(\xi, u)$, respectively, by the formulas (3.23), (3.22), and (3.21), where $g(h, \xi, \alpha) \equiv g_{h, \alpha}(\xi)$ is determined by Proposition 3.5.

Remark 3.8. In the case $\varkappa=k$, Theorem 3.7 holds under the assumptions $1 \leq p<(n-k) / k^{\prime}$ and $\mu>k^{\prime}$. 


\section{Inversion of the Dual Radon Transform for a Pair of AfFine Grassmannians}

Let us consider the dual Radon transform (2.9) which is well defined for every locally integrable function $\varphi$ on $G\left(n, k^{\prime}\right)$ and represents a locally integrable function on $G(n, k)$ by the formula

$$
\left(R^{*} \varphi\right)(\tau) \equiv\left(R^{*} \varphi\right)(\xi, u)=\int_{S O(n-k)} \varphi\left(g_{\xi} \rho \mathbb{R}^{k^{\prime}}+u\right) d \rho
$$

see Lemma 2.2. Our aim is to reconstruct $\varphi$ from $R^{*} \varphi$.

4.1. The Dual Radon Transform of Quasi-Radial Functions. In this subsection we consider the case when $\varphi$ is a locally integrable quasiradial function on $G\left(n, k^{\prime}\right)$. By Definition 3.1, there exists a function $\varphi_{0} \equiv \varphi_{0}(\eta, t)$ on $G_{n, k^{\prime}} \times \mathbb{R}_{+}$such that $\varphi(\eta, v)=\varphi_{0}(\eta,|v|)$ and

$$
\int_{0}^{a} t^{n-k^{\prime}-1} d t \int_{G_{n, k^{\prime}}}\left|\varphi_{0}(\eta, t)\right| d \eta<\infty \quad \forall a>0 .
$$

Our aim is to show that averaging $\left(R^{*} \varphi\right)(\xi, u)$ over all $u \in \xi^{\perp}$ at a distance $r$ from the origin yields a decomposition of $R^{*} \varphi$ into a tensor product of two invertible operators, namely, the Erdélyi-Kober type operator and the dual Funk-Radon transform for a pair of compact Grassmannians. We will be using the same notations for the coordinate planes as in (2.2)-(2.4).

Let $g_{\xi} \in S O(n)$ be a rotation satisfying $g_{\xi} \mathbb{R}^{k}=\xi$. We set

$$
\left(M_{\xi} f\right)(r)=\int_{S O(n-k)}\left(f \circ g_{\xi}\right)\left(\mathbb{R}^{k}, r \gamma e_{n}\right) d \gamma, \quad r>0
$$

where $S O(n-k)$ is the isotropy subgroup of the coordinate plane $\mathbb{R}^{n-k}$; see (2.3). Using (4.1) and setting $\varphi_{\xi}=\varphi \circ g_{\xi}$, we formally obtain

$$
\begin{aligned}
\left(M_{\xi} R^{*} \varphi\right)(r) & =\int_{S O(n-k)}\left(R^{*} \varphi \circ g_{\xi}\right)\left(\mathbb{R}^{k}, r \gamma e_{n}\right) d \gamma \\
& =\int_{S O(n-k)} d \gamma \int_{S O(n-k)} \varphi_{\xi}\left(\rho \mathbb{R}^{k^{\prime}}+r \gamma e_{n}\right) d \rho .
\end{aligned}
$$


This gives

$$
\begin{aligned}
\left(M_{\xi} R^{*} \varphi\right)(r) & =\int_{S O(n-k)} d \gamma \int_{S O(n-k)} \varphi_{\xi}\left(\rho \mathbb{R}^{k^{\prime}}, r \operatorname{Pr}_{\rho \mathbb{R}^{n-k^{\prime}}} \gamma e_{n}\right) d \rho \\
& =\int_{S O(n-k)} d \rho \int_{S O(n-k)} \varphi_{\xi}\left(\rho \mathbb{R}^{k^{\prime}}, r \rho \operatorname{Pr}_{\mathbb{R}^{n-k^{\prime}}} \rho^{T} \gamma e_{n}\right) d \gamma \\
& =\frac{1}{\sigma_{n-k}} \int_{S O(n-k)} d \rho \int_{S^{n-k-1}} \varphi_{\xi}\left(\rho \mathbb{R}^{k^{\prime}}, r \rho \operatorname{Pr}_{\mathbb{R}^{n-k^{\prime}}} \sigma\right) d \sigma
\end{aligned}
$$

where $S^{n-k-1}$ denotes the unit sphere in $\mathbb{R}^{n-k}$ and $\operatorname{Pr}_{\mathbb{R}^{n-k^{\prime}}}$ stands for the orthogonal projection onto $\mathbb{R}^{n-k^{\prime}}$. Passing to bi-spherical coordinates

$$
\sigma=\alpha \cos \psi+\beta \sin \psi, \quad \alpha \in S^{k^{\prime}-k-1}, \quad \beta \in S^{n-k^{\prime}-1},
$$

where $S^{k^{\prime}-k-1}$ and $S^{n-k^{\prime}-1}$ denote the unit spheres in $\mathbb{R}^{k^{\prime}-k}$ and $\mathbb{R}^{n-k^{\prime}}$, respectively (see, e.g. [15, Lemma 1.38]), we continue

$$
\begin{aligned}
\left(M_{\xi} R^{*} \varphi\right)(r) & =\frac{1}{\sigma_{n-k}} \int_{S O(n-k)} d \rho \int_{0}^{\pi / 2} \cos ^{k^{\prime}-k-1} \psi \sin ^{n-k^{\prime}-1} \psi d \psi \\
& \times \int_{S^{k^{\prime}-k-1}} d \alpha \int_{S^{n-k^{\prime}-1}} \varphi_{\xi}\left(\rho \mathbb{R}^{k^{\prime}},(\rho \beta) r \sin \psi\right) d \beta \\
& =\frac{\sigma_{k^{\prime}-k-1} \sigma_{n-k^{\prime}-1}}{\sigma_{n-k}} \int_{0}^{1} s^{n-k^{\prime}-1}\left(1-s^{2}\right)^{\left(k^{\prime}-k\right) / 2-1} d s \\
& \times \int_{S O(n-k)} \varphi_{\xi}\left(\rho \mathbb{R}^{k^{\prime}},\left(\rho e_{n}\right) r s\right) d \rho .
\end{aligned}
$$

Changing variables, we obtain

$$
\begin{aligned}
\left(M_{\xi} R^{*} \varphi\right)(r) & =\frac{\sigma_{k^{\prime}-k-1} \sigma_{n-k^{\prime}-1}}{r^{n-k-2} \sigma_{n-k}} \int_{0}^{r} t^{n-k^{\prime}-1}\left(r^{2}-t^{2}\right)^{\left(k^{\prime}-k\right) / 2-1} d t \\
& \times \int_{\eta \supset \xi} \varphi\left(\eta, t g_{\eta} e_{n}\right) d_{\xi} \eta
\end{aligned}
$$

$g_{\eta}$ being an orthogonal transformation that takes $\mathbb{R}^{k^{\prime}}$ to $\eta$. Since $\varphi$ is quasi-radial, it follows that $\varphi\left(\eta, t g_{\eta} e_{n}\right) \equiv \varphi_{0}(\eta, t)$. Then the integral in (4.6) is the dual Funk-Radon transform $(2.40)$ of $\varphi_{0}(\cdot, t)$ and we can 
write

$$
\begin{gathered}
\left(M_{\xi} R^{*} \varphi\right)(r)=\frac{c}{r^{n-k-2}}\left(\mathcal{F}_{(n)}^{*} \Psi_{r}\right)(\xi), \quad c=\frac{\pi^{\left(k^{\prime}-k\right) / 2} \sigma_{n-k^{\prime}-1}}{\sigma_{n-k}}, \\
\Psi_{r}(\eta)=\left(I_{+, 2}^{\left(k^{\prime}-k\right) / 2}\left[\psi_{(\cdot)}(\eta)\right]\right)(r), \quad \psi_{t}(\eta)=t^{n-k^{\prime}-2} \varphi_{0}(\eta, t) .
\end{gathered}
$$

To make the formal derivation of (4.7) rigorous, we need to show that the right-hand side of (4.7) exists in the Lebesgue sense when $\varphi$ is replaced by $|\varphi|$. To this end, it suffices to assume that $\varphi(\eta, v) \equiv$ $\varphi_{0}(\eta,|v|)$ is nonnegative and prove the following statements.

(a) The function $t \rightarrow t \psi_{t}(\eta)$ is locally integrable on $\mathbb{R}_{+}$for almost all $\eta \in G_{n, k^{\prime}}$

(b) $\Psi_{r} \in L^{1}\left(G_{n, k^{\prime}}\right)$ for almost all $r>0$.

The statement (a) follows from (4.2). To prove (b), owing to (4.8), we have

$$
\int_{G_{n, k^{\prime}}} \Psi_{r}(\eta) d \eta=\left(I_{+, 2}^{\left(k^{\prime}-k\right) / 2} \tilde{\psi}\right)(r),
$$

where $\tilde{\psi}(t)=t^{n-k^{\prime}-2} \int_{G_{n, k^{\prime}}} \varphi_{0}(\eta, t) d \eta$. By $(4.2), t \tilde{\psi}(t)$ is locally integrable on $\mathbb{R}_{+}$and therefore, by Lemma 2.5(i), the expression (4.9) is finite for almost all $r>0$. This gives (b).

The equalities (4.7) and (4.8) reduce the inversion problem for $R^{*} \varphi$ to inversion of $\mathcal{F}_{(n)}^{*}$ and $I_{+, 2}^{\left(k^{\prime}-k\right) / 2}$.

Theorem 4.1. Let $f=R^{*} \varphi$, where $\varphi \equiv \varphi_{0}(\eta,|v|)$ is a locally integrable quasi-radial function on $G\left(n, k^{\prime}\right), 0<k<k^{\prime}<n, k+k^{\prime} \geq n$. Then for almost all $\eta \in G_{n, k^{\prime}}$ and almost all $t>0$,

$$
\begin{aligned}
\varphi_{0}(\eta, t) & =c^{-1} t^{k^{\prime}+2-n}\left(\mathcal{D}_{+, 2}^{\left(k^{\prime}-k\right) / 2}\left[\Psi_{(\cdot)}(\eta)\right]\right)(t), \\
\Psi_{r}(\eta) & =r^{n-k-2}\left(\left(\mathcal{F}_{(n)}^{*}\right)^{-1}\left[\left(M_{(\cdot)} f\right)(r)\right]\right)(\eta),
\end{aligned}
$$

where $c$ is a constant from $(4.7), \mathcal{D}_{+, 2}^{\left(k^{\prime}-k\right) / 2}$ and $\left(\mathcal{F}_{(n)}^{*}\right)^{-1}$ being defined by (2.20) and (2.52), respectively.

Remark 4.2. It is known that both $R$ and $R^{*}$ take radial functions to radial ones on the corresponding affine Grassmannians [10]. For quasi-radial functions the situation is different. In Subsection 3.1 it was shown that the operator $R$ takes quasi-radial functions on $G(n, k)$ to quasi-radial functions on $G\left(n, k^{\prime}\right)$. A similar result for $R^{*}$ may not be true. Let, for example, $k=1$ and $k^{\prime}=n-1$. Then (2.10) with 
$\xi=\mathbb{R}^{1}$ and $u=e_{m}, m>1$, yields

$$
\begin{aligned}
\left(R^{*} \varphi\right)\left(\mathbb{R}^{1}, e_{m}\right) & =\int_{S O(n-1)} \varphi\left(\rho e_{n}^{\perp}+\operatorname{Pr}_{\rho e_{n}} e_{m}\right) d \rho \\
& =\int_{S O(n-1)} \varphi\left(\left(\rho e_{n}\right)^{\perp}+\left(\rho e_{n}\right)\left(\rho e_{n} \cdot e_{m}\right)\right) d \rho \\
& =\int_{S^{n-2}} \varphi\left(\sigma^{\perp}+\sigma\left(\sigma \cdot e_{m}\right)\right) d_{*} \sigma
\end{aligned}
$$

where $S^{n-2}$ stands for the unit sphere in $e_{1}^{\perp}$. Now we choose $\varphi=\varphi_{0}$, where $\varphi_{0}(\eta, v)=\left|v \cdot e_{2}\right|$. The orthogonal complement to $\eta$ is onedimensional and therefore, the only orthogonal transformation that keeps $\eta$ fixed is the reflection $v \rightarrow-v$. It follows that $\varphi_{0}$ is quasi-radial. Assuming, for simplicity, $n=3$, we have the following expressions for $m=2$ and $m=3$ :

$$
\begin{gathered}
\left(R^{*} \varphi_{0}\right)\left(\mathbb{R}^{1}, e_{2}\right)=\int_{S^{1}}\left(\sigma \cdot e_{2}\right)^{2} d_{*} \sigma=\frac{1}{2 \pi} \int_{0}^{2 \pi} \cos ^{2} \theta d \theta=\frac{1}{2}, \\
\left(R^{*} \varphi_{0}\right)\left(\mathbb{R}^{1}, e_{3}\right)=\int_{S^{1}}\left|\left(\sigma \cdot e_{2}\right)\left(\sigma \cdot e_{3}\right)\right| d_{*} \sigma=\frac{1}{2 \pi} \int_{0}^{2 \pi}|\cos \theta \sin \theta| d \theta=\frac{1}{\pi} .
\end{gathered}
$$

Thus $\left(R^{*} \varphi_{0}\right)\left(\mathbb{R}^{1}, e_{2}\right) \neq\left(R^{*} \varphi_{0}\right)\left(\mathbb{R}^{1}, e_{3}\right)$, which means that $R^{*} \varphi_{0}$ is not quasi-radial.

4.2. The Dual Radon Transform. The general case. By Lemma 2.4, the dual Radon transform $f=R^{*} \varphi$ that takes functions $\varphi \in$ $L_{k+1}^{1}\left(G\left(n, k^{\prime}\right)\right)$ to functions on $G(n, k)$ is injective if and only if $k+k^{\prime} \geq$ $n-1$. To reconstruct $\varphi$ from $f$, we use some ideas from [10, Section 4] according to which $R^{*}$ expresses through a certain auxiliary Radon transform $\mathfrak{R}$ that takes functions on $G\left(n, n-k^{\prime}-1\right)$ to functions on $G(n, n-k-1)$. The new transform $\mathfrak{R}$ can be explicitly inverted, e.g., as in Section 3. Thus we shall arrive at explicit inversion of $R^{*}$.

Let us proceed to details. For $\tau \equiv \tau(\xi, u) \in G(n, k)$, with $u \neq 0$, we denote by $\{\tau\} \in G_{n, k+1}$ the smallest linear subspace containing $\tau$, and set

$\tilde{\xi}=\{\tau\}^{\perp} \in G_{n, n-k-1}, \quad \tilde{u}=-\frac{u}{|u|^{2}} \in \tilde{\xi}^{\perp}, \quad \tilde{\tau} \equiv \tilde{\tau}(\tilde{\xi}, \tilde{u}) \in G(n, n-k-1)$.

Consider the Kelvin-type mapping

$$
G(n, k) \ni \tau \stackrel{\nu}{\longrightarrow} \tilde{\tau} \in G(n, n-k-1) .
$$


Clearly, $\nu(\nu(\tau))=\tau$ and $|\tau|=|\tilde{\tau}|^{-1}$ (see Notation). In a similar way, for $\zeta \equiv \zeta(\eta, v) \in G\left(n, k^{\prime}\right), v \neq 0$, we denote

$$
\tilde{\eta}=\{\zeta\}^{\perp} \in G_{n, n-k^{\prime}-1}, \quad \tilde{v}=-\frac{v}{|v|^{2}} \in \tilde{\eta}^{\perp}, \quad \tilde{\zeta} \equiv \tilde{\zeta}(\tilde{\eta}, \tilde{v}) \in G\left(n, n-k^{\prime}-1\right),
$$

so that

$$
G\left(n, k^{\prime}\right) \ni \zeta \stackrel{\nu}{\longrightarrow} \tilde{\zeta} \in G\left(n, n-k^{\prime}-1\right) .
$$

Definition 4.3. Let $R: f(\tau) \rightarrow(R f)(\zeta)$ be the Radon transform (2.6) that takes functions on $G(n, k)$ to functions on $G\left(n, k^{\prime}\right), k^{\prime}>k$. If $\tilde{\tau}=\nu(\tau) \in G(n, n-k-1)$ and $\tilde{\zeta}=\nu(\zeta) \in G\left(n, n-k^{\prime}-1\right)$, then the associated Radon transform $\tilde{f}(\tilde{\zeta}) \rightarrow(\Re \tilde{f})(\tilde{\tau})$ that integrates $\tilde{f}$ over all $\tilde{\zeta}$ in $\tilde{\tau}$ is called quasi-orthogonal to $R$.

Theorem 4.4. [10, Theorem 5.5] Let $0 \leq k<k^{\prime}<n$. For a function $\varphi$ on $G\left(n, k^{\prime}\right)$, we denote

$$
(A \varphi)(\tilde{\zeta})=|\tilde{\zeta}|^{k-n} \varphi\left(\nu^{-1}(\tilde{\zeta})\right), \quad \tilde{\zeta} \in G\left(n, n-k^{\prime}-1\right) .
$$

(i) The following relation holds

$$
\int_{G\left(n, k^{\prime}\right)} \frac{\varphi(\zeta) d \zeta}{\left(1+|\zeta|^{2}\right)^{(k+1) / 2}}=\frac{\sigma_{n-k^{\prime}-1}}{\sigma_{k^{\prime}}} \int_{G\left(n, n-k^{\prime}-1\right)} \frac{(A \varphi)(\tilde{\zeta}) d \tilde{\zeta}}{\left(1+|\tilde{\zeta}|^{2}\right)^{(k+1) / 2}}
$$

provided that either side of this equality exists in the Lebesgue sense.

(ii) If at least one of the integrals in (4.13) is finite, then

$$
\left(R^{*} \varphi\right)(\tau)=c|\tau|^{k^{\prime}-n}(\Re A \varphi)(\nu(\tau)), \quad c=\frac{\sigma_{n-k^{\prime}-1}}{\sigma_{n-k-1}} .
$$

Theorem 4.4 paves the way to reconstruction of $\varphi$ from $f=R^{*} \varphi$. Indeed, we write $(4.14)$ as $(\Re A \varphi)(\nu(\tau))=c^{-1}|\tau|^{n-k^{\prime}}\left(R^{*} \varphi\right)(\tau)$ or

$$
(\Re A \varphi)(\tilde{\tau}))=c^{-1}|\tilde{\tau}|^{k^{\prime}-n} f\left(\nu^{-1}(\tilde{\tau})\right) .
$$

Inverting $\mathfrak{R}$ as in Section 3, we formally obtain

$$
\varphi(\zeta)=|\zeta|^{k-n}\left(\Re^{-1} f_{1}\right)(\nu(\zeta)), \quad f_{1}(\tilde{\tau})=c^{-1}|\tilde{\tau}|^{k^{\prime}-n} f\left(\nu^{-1}(\tilde{\tau})\right) .
$$

To make this reasoning rigorous, we need to choose a suitable class of functions $\varphi$ that guarantees applicability of (4.14) and Theorem 3.7.

For $1 \leq p<\infty$, we denote

$$
\tilde{L}^{p}\left(G\left(n, k^{\prime}\right)\right)=\left\{\varphi: \int_{G\left(n, k^{\prime}\right)}|\zeta|^{(n-k) p-n-1}|\varphi(\zeta)|^{p} d \zeta<\infty\right\} .
$$


For $\mu \in \mathbb{R}$, let $\tilde{C}_{\mu}\left(G\left(n, k^{\prime}\right)\right)$ be the space of all functions $\varphi$ which are continuous on the set of all $k^{\prime}$-planes $\zeta \subset \mathbb{R}^{n}$ not passing through the origin and satisfy the following condition:

$$
\begin{cases}|\zeta|^{n-k-\mu} \varphi(\zeta)=O(1) & \text { if }|\zeta| \rightarrow 0 \\ |\zeta|^{n-k} \varphi(\zeta) \rightarrow \text { const } & \text { if }|\zeta| \rightarrow \infty\end{cases}
$$

The choice of these classes of functions is motivated by the following lemma.

\section{Lemma 4.5.}

(i) For any $1 \leq p<\infty$, the relations $\varphi \in \tilde{L}^{p}\left(G\left(n, k^{\prime}\right)\right)$ and $A \varphi \in$ $L^{p}\left(G\left(n, n-k^{\prime}-1\right)\right)$ are equivalent. If $1 \leq p<\left(k^{\prime}+1\right) /\left(k^{\prime}-k\right)$, then the Radon transform $\mathfrak{R} A \varphi$ exists in the Lebesgue sense and

$$
\int_{G\left(n, k^{\prime}\right)} \frac{|\varphi(\zeta)| d \zeta}{\left(1+|\zeta|^{2}\right)^{(k+1) / 2}}<\infty .
$$

(ii) For any $\mu \in \mathbb{R}$, the relations $\varphi \in \tilde{C}_{\mu}\left(G\left(n, k^{\prime}\right)\right)$ and $A \varphi \in$ $C_{\mu}\left(G\left(n, n-k^{\prime}-1\right)\right)$ are equivalent. If $\mu>k^{\prime}-k$, then the Radon transform $(\Re A \varphi)(\tilde{\tau})$ is finite for every $\tilde{\tau} \in G(n, n-k-1)$ and (4.19) holds.

Proof. (i) To prove the first statement, we observe that

$$
\|A \varphi\|_{p}^{p}=\int_{G\left(n, n-k^{\prime}-1\right)}|\tilde{\zeta}|^{(k-n) p}\left|\varphi\left(\nu^{-1}(\tilde{\zeta})\right)\right|^{p} d \tilde{\zeta}=\int_{G\left(n, n-k^{\prime}-1\right)} \frac{(A \psi)(\tilde{\zeta}) d \tilde{\zeta}}{\left(1+|\tilde{\zeta}|^{2}\right)^{(k+1) / 2}}
$$

where

$$
\psi(\zeta)=\left(1+|\zeta|^{2}\right)^{(k+1) / 2}|\zeta|^{(n-k) p-n-1}|\varphi(\zeta)|^{p}
$$

Hence, by (4.13),

$$
\begin{aligned}
\frac{\sigma_{n-k^{\prime}-1}}{\sigma_{k^{\prime}}}\|A \varphi\|_{p}^{p} & =\int_{G\left(n, k^{\prime}\right)} \frac{\psi(\zeta) d \zeta}{\left(1+|\zeta|^{2}\right)^{(k+1) / 2}} \\
& =\int_{G\left(n, k^{\prime}\right)}|\zeta|^{(n-k) p-n-1}|\varphi(\zeta)|^{p} d \zeta,
\end{aligned}
$$

as desired. The existence of $\Re A \varphi$ follows from Lemma $2.2 ;(4.19)$ holds by Hölder's inequality.

(ii) The proof of the first statement and the finiteness of the righthand side of (4.13) for $\mu>k^{\prime}-k$ is straightforward. Hence the lefthand side of (4.13) is finite. The existence of $\Re A \varphi$ follows from Lemma 2.2 . 
Now we are ready to formulate the main inversion result for $R^{*}$ that follows from (4.14) and Theorem 3.7. Note that application of this theorem leads to some additional restrictions on the classes of functions in comparison with those in Lemma 4.5.

Theorem 4.6. Let

$$
1 \leq k<k^{\prime} \leq n-1, \quad k+k^{\prime} \geq n-1, \quad n-k^{\prime}-1 \leq \varkappa<k+1,
$$

and suppose that $f=R^{*} \varphi$. If

$$
\varphi \in \tilde{L}^{p}\left(G\left(n, k^{\prime}\right)\right), \quad 1 \leq p<\frac{k^{\prime}+1}{k^{\prime}-k+\varkappa},
$$

or

$$
\varphi \in \tilde{C}_{\mu}\left(G\left(n, k^{\prime}\right)\right), \quad \mu>k^{\prime}-k+\varkappa,
$$

then $\varphi$ can be reconstructed from $f$ by the formula (4.16) in which $\mathfrak{R}^{-1}$ defined in accordance with Theorem 3.7.

Remark 4.7. The case $\varkappa=n-k^{\prime}-1$ in Theorem 4.6 might be of particular interest. In this case Theorem 4.6 holds under the assumptions

$$
1 \leq p<\frac{k^{\prime}+1}{n-k-1}, \quad \mu>n-k-1 .
$$

\section{Concluding Remarks}

In the present paper we suggested several straightforward inversion algorithms for the Radon transform and its dual on affine Grassmannians $G(n, k)$ and $G\left(n, k^{\prime}\right)$. In particular, the Gonzalez-Kakehi Fourier inversion method for Schwartz functions [3] was extended to more general $L^{p}$ and continuous functions and arbitrary parity of $k$ and $k^{\prime}$. To this end, we have replaced the Fourier transform by the relevant Radon-John transform. The price for this improvement is an additional parameter $\varkappa$ that makes the classes of functions not optimal. The question of how to eliminate this parameter (without using stereographic projection, as in [10]), remains open and requires new ideas.

Acknowledgements. This work was begun when the second-named author was visiting the Department of Mathematics, Louisiana State University, in 2014-2015. He would like to express his gratitude to the administration of this Department for the hospitality.

\section{REFERENCES}

[1] I. M. Gelfand, S. G. Gindikin and M. I. Graev, Selected Topics in Integral Geometry, Translations of Mathematical Monographs, AMS, Providence, Rhode Island, 2003. 
[2] F. B. Gonzalez, John's equation and the plane-to-line transform on $\mathbb{R}^{3}$, Harmonic analysis and integral geometry (Safi, 1998), 1-7, Chapman \& Hall/CRC Res. Notes Math., 422, Chapman \& Hall/CRC, 2001.

[3] F. B. Gonzalez and T. Kakehi, Pfaffian systems and Radon transforms on affine Grassmann manifolds, Math. Ann. (2) 326 (2003), 237-273.

[4] Dual Radon transforms on affine Grassmann manifolds, Trans. Amer. Math. Soc. (10) 356 (2004), 4161-4180.

[5] M. I. Graev, A problem of integral geometry related to a triple of Grassmann manifolds, Funct. Anal. and its Appl., 34 (2000), 299-301.

[6] E. Grinberg and B. Rubin, Radon inversion on Grassmannians via Gårding-Gindikin fractional integrals, Annals of Math. 159 (2004), 809843.

[7] S. Helgason, Groups and Geometric Analysis: Integral Geometry, Invariant Differential Operators, and Spherical Functions, Academic Press, 2000.

[8] _ Integral Geometry and Radon Transform, Springer, New YorkDordrecht-Heidelberg-London, 2011.

[9] T. Kakehi, Integral geometry on Grassmann manifolds and calculus of invariant differential operators, J. Funct. Anal. 168 (1999), 1-45.

[10] B. Rubin, Radon transforms on affine Grassmannians, Trans. Amer. Math. Soc. 356 (2004), 5045-5070.

[11] — Reconstruction of functions from their integrals over $k$ dimensional planes, Israel J. of Math. 141 (2004), 93-117.

[12] _ Funk, cosine, and sine transforms on Stiefel and Grassmann manifolds, J. of Geometric Analysis (3) 23 (2013), 1441-1497.

[13] - Weighted norm inequalities for $k$-plane transforms, Proc. Amer. Math. Soc. 142 (2014), 3455-3467.

[14] - On the Funk-Radon-Helgason inversion method in integral geometry, Contemp. Math. 599 (2013), 175-198.

[15] _ Introduction to Radon transforms: with elements of fractional calculus and harmonic analysis, Encyclopedia of Mathematics and its Applications, 160, Cambridge University Press, 2015.

[16] B. Rubin and Yingzhan Wang, On Radon transforms between lines and hyperplanes, arXiv:1601.03826.

[17] R. S. Strichartz, Harmonic analysis on Grassmannian bundles, Trans. Amer. Math. Soc. (1) 296 (1986), 387-409.

[18] Genkai Zhang, Radon transform on real, complex, and quaternionic Grassmannians, Duke Math. J. 138 (2007), 137-160.

Department of Mathematics, Louisiana State University, Baton Rouge, LA, 70803 USA

E-mail address: borisr@math.lsu.edu

School of Mathematics and Information Science, Guangzhou UniVersity, Guangzhou 510006, China;

E-mail address: wyzde@gzhu.edu.cn 